\title{
Observation of Vortices and Columnar Defects by Using Lorentz Microscopy
}

${ }^{1,2,6}$ H. Kasai, ${ }^{1}$ O. Kamimura, ${ }^{1,2,6}$ T. Matsuda, ${ }^{1,2,6} \mathrm{~K}$. Harada, ${ }^{1,2,6}$ A. Tonomura, ${ }^{3} \mathrm{~S}$. Okayasu, ${ }^{3}$ M. Sasase, ${ }^{4,6}$ Y. Nakayama, ${ }^{4,6}$ J. Shimoyama, ${ }^{4,6}$ K. Kishio, ${ }^{5,6}$ T. Hanaguri and ${ }^{5,6}$ K. Kitazawa

1) Advanced Research Laboratory, Hitachi, Ltd., Hatoyama, Saitama 350-0395, Japan

2) FRS, The Institute of Physical and Chemical Research (RIKEN), Wako, Saitama 351-0198, Japan

3) Japan Atomic Energy Research Institute (JAERI), Tokai, Ibaraki 319-1195, Japan

4) Department of Applied Chemistry, University of Tokyo, Tokyo 113-8656, Japan

5) Department of Advanced Materials Science, University of Tokyo, Tokyo 113-0033, Japan

6) SORST, Japan Science and Technology Corporation (JST), Chuo-ku, Tokyo 103-0027, Japan

The understanding of the pinning mechanism for individual vortices (magnetic flux lines) in a Type-II superconductor is important for developing practical applications because the vortex flow generates heat and breaks down the superconducting state. The interaction between vortices and pinning centers, especially columnar defects, has been the subject of numerous studies. No means, however, have been available to obtain information about whether each single vortex inside a material is actually pinned along columnar defects or not. Recently, the 1-MV field-emission electron microscope [1] allowed us to observe vortices in $\mathrm{Bi}_{2} \mathrm{Sr}_{2} \mathrm{CaCu}_{2} \mathrm{O}_{8+\delta}(\mathrm{Bi}-2212)$ films with high contrast whose thickness of about $400 \mathrm{~nm}$ [2]. Here we report the study to obtain direct information about the vortex structure inside the material by Lorentz microscopy.

Thin films with a thickness of about $400 \mathrm{~nm}$ and a $100 \times 100 \mu \mathrm{m}^{2}$ area as specimens were cleaved from a single crystalline of Bi-2212 grown by the standard floating-zone technique [3]. Columnar defects with a density of $2.5 \times 10^{7}$ ions $/ \mathrm{cm}^{2}(0.5 \mathrm{mT})$ the angles of which were $0^{\circ}$ and $70^{\circ}$ tilted to the c-axis of Bi-2212 were introduced by irradiation of $240 \mathrm{MeV} \mathrm{Au}{ }^{15+}$ ions at the tandem accelerator of the Japan Atomic Energy Research Institute.

Figure 1(a) is a high-resolution micrograph recorded in the [001] direction of a columnar defect that was produced a few degrees off the c-axis of the crystal [4]. Figure 1(b) shows the random distribution of the columnar defects. The defects, corresponding to the black spots in the figure, are rather difficult to observe in low magnified images. However, they are easier to observe even by low magnification when tilted because they are elongated as line shapes along the ion trajectory shown in Fig. 1(c).

Next, the prepared thin film was set on a low-temperature holder tilted $30^{\circ}$ to an electron beam in order to detect the vortex contrast in Lorentz micrographs. Figure 2 shows a schematic of the experimental setup of the vortex observation. A magnetic field can be applied in any direction to the specimen. Both vortices trapped at columnar defects and free from the defects were observed simultaneously. In the Lorentz micrograph, globule-like black and white contrast features correspond to a single vortex.

Figure 3(a) is an infocus electron micrograph at $30 \mathrm{~K}$ and $0.5 \mathrm{mT}$ that shows the columnar defects indicated by arrows. Figure 3(b) is Lorentz micrograph the defocus of which is $500 \mathrm{~mm}$. The vortex images can be roughly divided into two-types: one with higher contrast and the other with lower contrast. Lower contrast vortices located at the columnar defects are indicated by arrows in Fig. 3(a). We concluded that the weak contrast images correspond to vortices pinned along the columnar defects, whereas high-contrast images correspond to unpinned vortices with a direction perpendicular to the specimen surface.

We found the surprising phenomena of pinned vortices at the columnar defects. The weak contrast of the 
pinned vortices remained unchanged when the temperature was increased. However, when the temperature was decreased to below $12 \mathrm{~K}$, the weak contrast images were changed to circular vortex images, the same as those surrounding unpinned vortices. We considered that the pinned vortices began to "stand up" perpendicularly to the film plane at lower temperatures.

These findings regarding the vortex phenomena can be explained microscopically as follows: there are two kinds of pinning centers in the materials. One is columnar defect that are stronger at higher temperature and the other may be many atomic-size defects which become effective at lower temperatures. Then vortex configuration depends on the temperature. Detailed experiments and simulations are now in progress in order to confirm this explanation.

\section{References}

[1] T. Kawasaki et al., Appl. Phys. Lett. 76 (2000) 1342.

[2] A. Tonomura et al., Nature 412 (2001) 620.

[3] Y. Kotaka et al., Physica C 235-240 (1994) 1529.

[4] K. Harada et al., Phys. Rev. B 53 (1996) 9400.

[5] We are grateful to N. Hatano, G. Pozzi, M. Beleggia, N. Osakabe T. Yoshida, J. Masuko, F. Nori, T. Akashi and I. Matsui for their discussion and technical help.
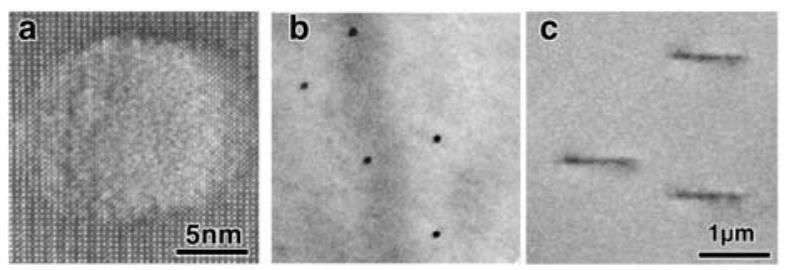

Figure 1(a) High resolution electron micrograph of columnar defect, (b) distribution of columnar defects, (c) columnar defect of $70^{\circ}$ tilted to c-axis of crystal.

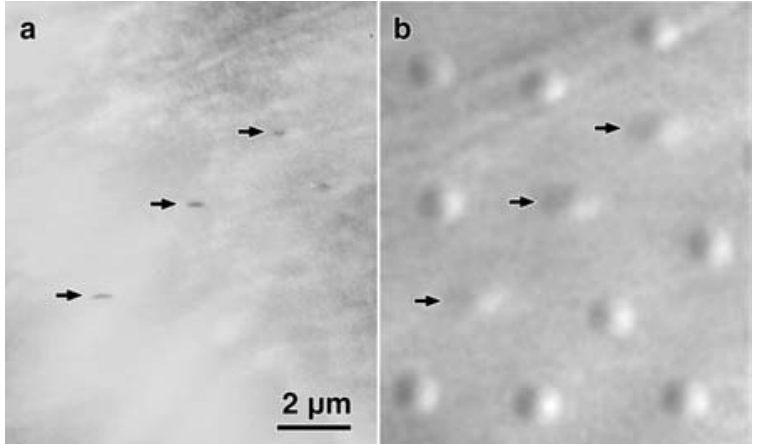

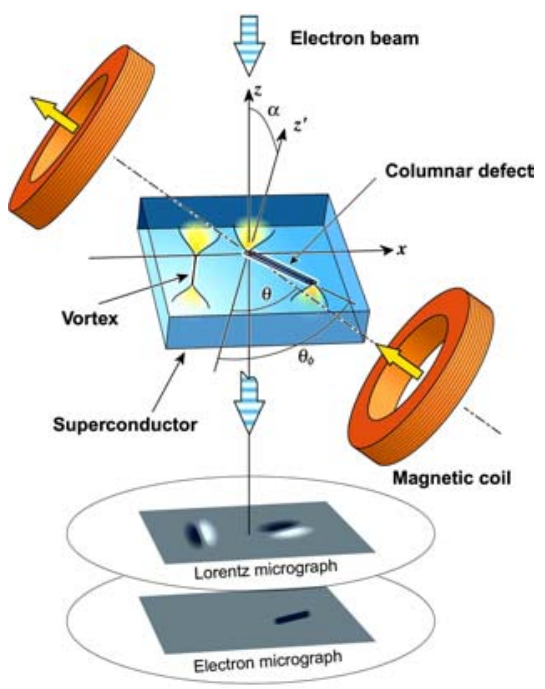

Figure 2 Experimental setup of vortex observation with columnar defect.

Figure 3(a) Electron micrograph, (b) Lorentz micrograph. Arrows show columnar defects. Vortices at defects are elongated and weak contrast images due to pinning along columns. 